\title{
Probing QCD with Jets, Photons and Weak Bosons at the LHC with ATLAS
}

\author{
Ben Cooper, on behalf of the ATLAS Collaboration \\ University College London, London, UK
}

\begin{abstract}
A summary of ATLAS measurements that probe "hard" QCD interactions in the protonproton collisions of the LHC are presented.
\end{abstract}

Keywords: atlas, qcd, jets

PACS: $10,12.38 . \mathrm{Qk}$

\section{INTRODUCTION}

The large datasets of $\sqrt{s}=7 \mathrm{TeV}$ proton-proton collisions collected by ATLAS detector [1] at the LHC provide the opportunity to probe short-distance strong interactions with a better precision and at a higher energy than has been possible before. Presented here is a short summary of the measurements made at ATLAS which test fixed order perturbative QCD and Monte Carlo predictions. All observables are (where appropriate) defined using anti- $\mathrm{k}_{T} \mathrm{R}=0.4$ and/or $\mathrm{R}=0.6$ jets [2], and are fully unfolded back to the stable particle-level. Due to space constraints only a very limited selection of the full results for certain measurements can be reported here.

\section{INCLUSIVE JETS AND DIJETS MEASUREMENTS}

A double-differential measurement of the inclusive cross-section for jet production as a function of $p_{\mathrm{T}}$ and rapidity has been made at ATLAS [3], for jets with rapidities extending to $|y|<4.4$. On the left of Fig. 1 we can see that good agreement between the measurement, NLOJET++ [4] and POWHEG [5] fixed order NLO predictions is observed, but there are significant differences in the success of the various POWHEG plus parton shower Monte Carlos in reproducing the data.

The ATLAS measurement of dijet production as a function of dijet invariant mass $\left(m_{12}\right)$ and $y^{*}=\left|y_{1}-y_{2}\right| / 2$ has recently been updated to use $4.8 \mathrm{fb}^{-1}$ [6], giving improved statistical precision at high masses. On the right of Fig. 1 one can see some tension between the measurement and the fixed order NLOJET++ prediction at high $m_{12}$, high $y^{*}$, with the NLOJET++ prediction apparently overestimating the measured cross-section.

Important measurements of the production of dijets with a central jet veto [7] and of the inclusive $b$-jet and dijet $b$-jet production [8] have also been made by ATLAS. 

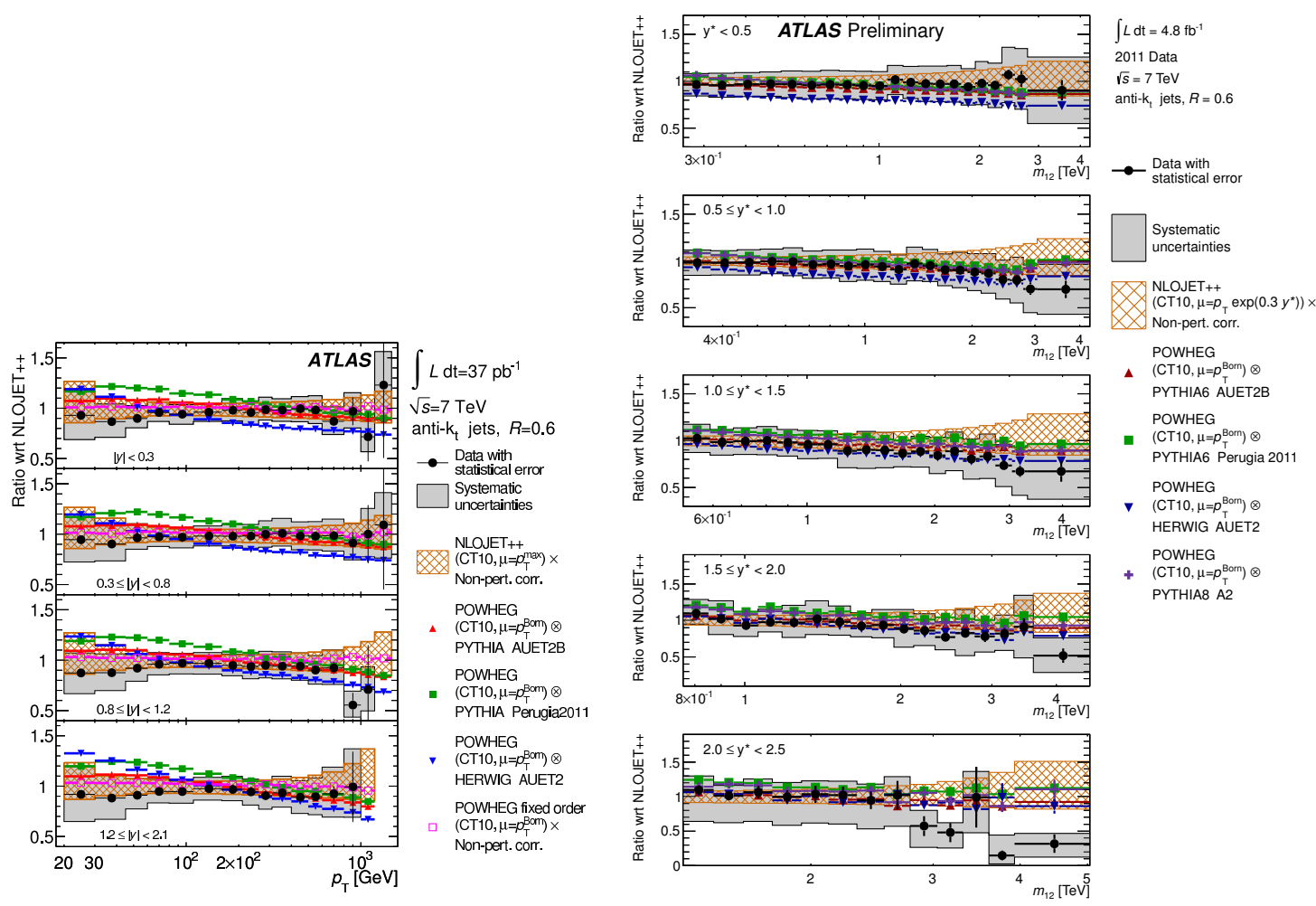

FIGURE 1. The ratio of the ATLAS measurement and various Monte Carlo predictions to the NLOJET++ prediction, on the left of the inclusive jet $p_{\mathrm{T}}$ spectra in different (selected) jet rapidity ranges [3], and on the right of the dijet invariant mass in different ranges of $y^{*}[6]$.

\section{MEASUREMENTS INVOLVING BOSONS}

ATLAS recently published an extensive study of the production of a $W$-boson in association with jets [9], including fully unfolded differential cross-sections as a function of many different variables; the number of jets, jet $p_{\mathrm{T}}, H_{\mathrm{T}}$, mass, rapidity and jet-jet angular separations. On the left of Fig. 2 we see important differences in the ability of various Monte Carlo generators to reproduce the ATLAS measured inclusive jet multiplicity ratio in $W$ plus jets events. A complementary measurement of $Z$ plus jets production has also been published by ATLAS [10].

ATLAS has also made detailed measurements of vector boson production in association with $b$-jets. On the right of Fig. 2 the measured cross-section for $W$ plus $\geq 1 b$-jet production as a function of the exclusive jet multiplicity is shown [11], and compared to the predictions of a fixed order NLO calculation and various Monte Carlo generators. Although consistent, there is a tension between the measurement and NLO prediction. A measurement of the cross-section for the production of $b$-jets in association with a $Z$-boson has also been made at ATLAS [12].

An alternative, more inclusive way to probe QCD predictions using vector bosons is to measure their transverse momenta. ATLAS has made measurements of the cross-section for the production of a vector boson as a function of it's transverse momentum for both 

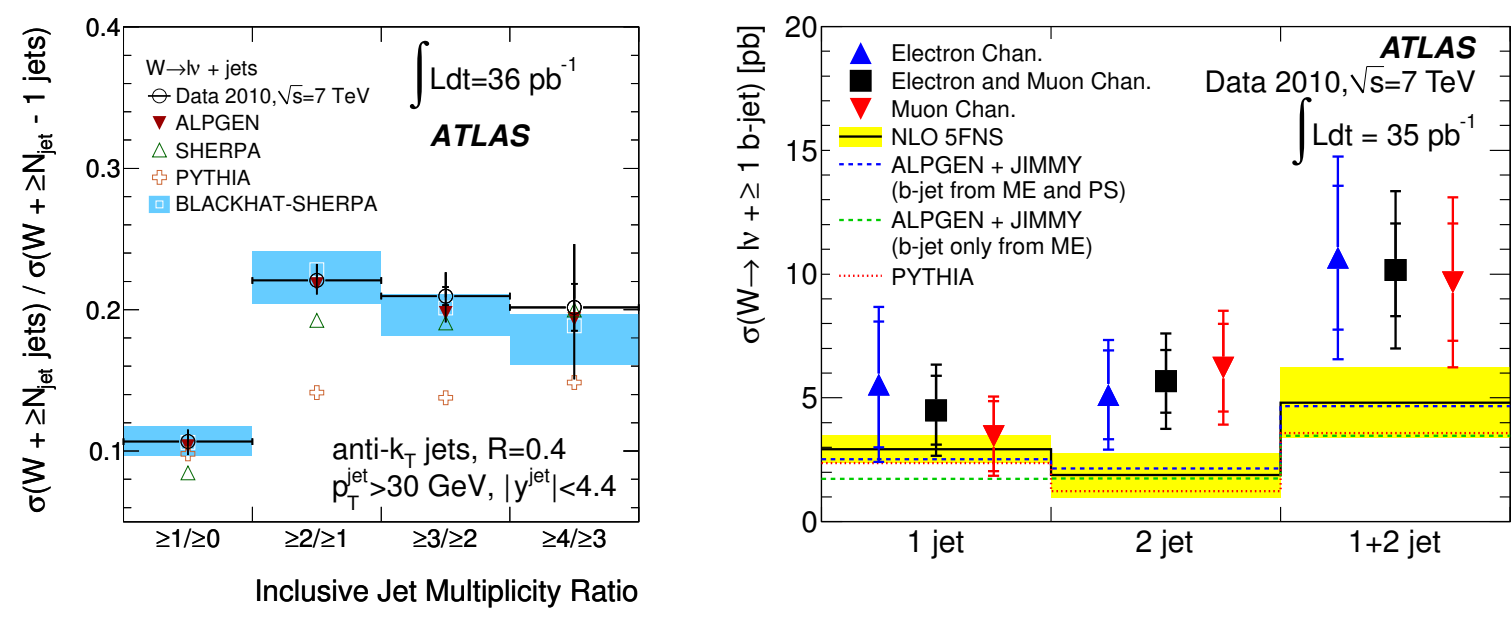

FIGURE 2. On the left the measured inclusive jet multiplicity ratio for $W$ plus jets production compared to various Monte Carlo predictions [9]. On the right, the measured cross-section for $W$ plus $\geq 1 b$-jet production in exclusive jet multiplicity bins [11], compared to NLO predictions.
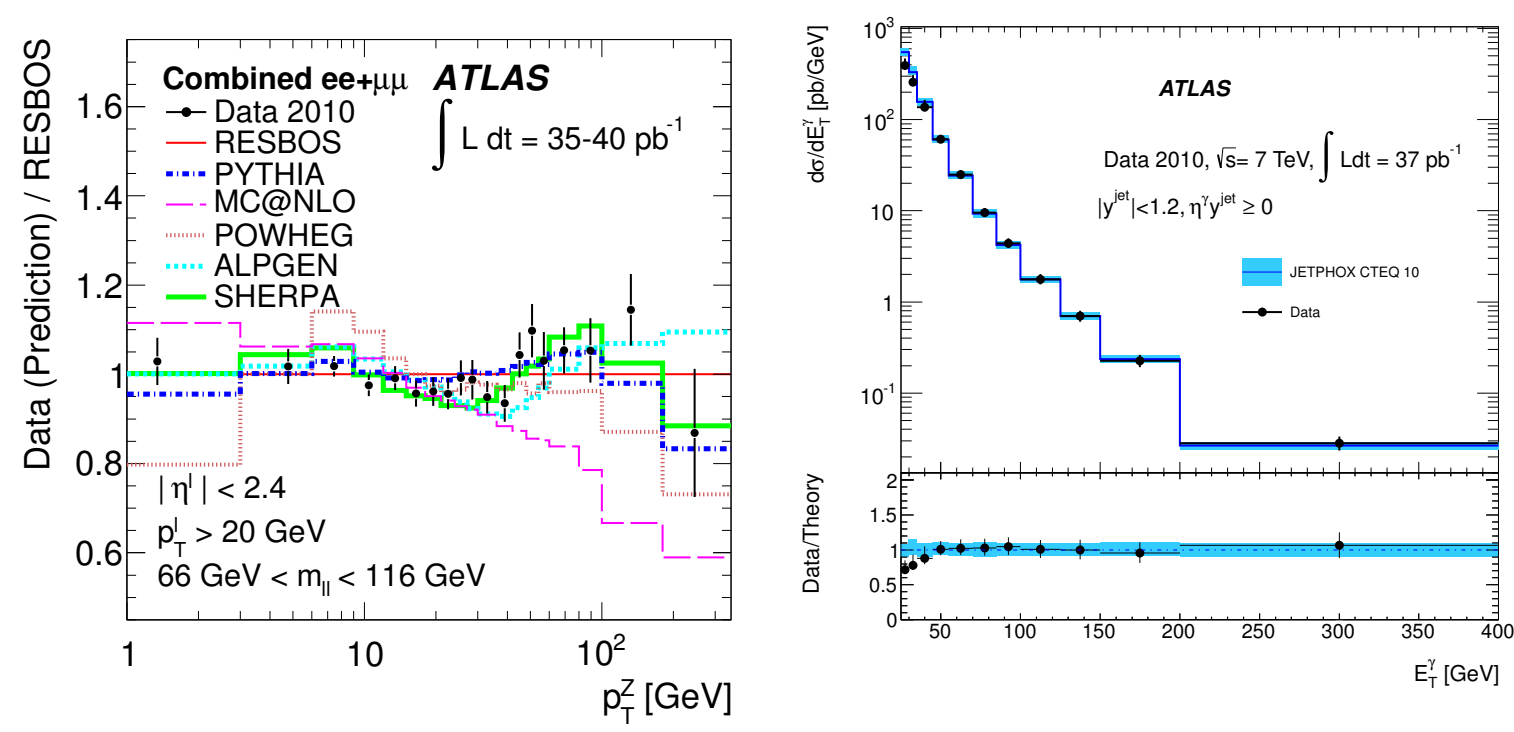

FIGURE 3. On the left, the ratio of the ATLAS measurement and various Monte Carlo predictions to the RESBOS prediction for the Z-boson transverse momentum [13]. On the right, the ATLAS measurement of the photon transverse energy spectra for photon plus jet events in a particular $\eta^{\gamma} y^{\text {jet }}$ angular configuration, compared to a NLO prediction [16].

$Z$ - and $W$-bosons separately [13, 14]. On the left of Fig. 3 we see that RESBOS [15], as well as the predictions of several other Monte Carlo generators, reproduces well the measured $Z$-boson $p_{\mathrm{T}}$ spectra.

Final states involving photons produced directly in the hard scatter can also be used as a probe of QCD. Recently, ATLAS published a differential cross-section measurement for the production of isolated photons in association with jets as a function of the photon 

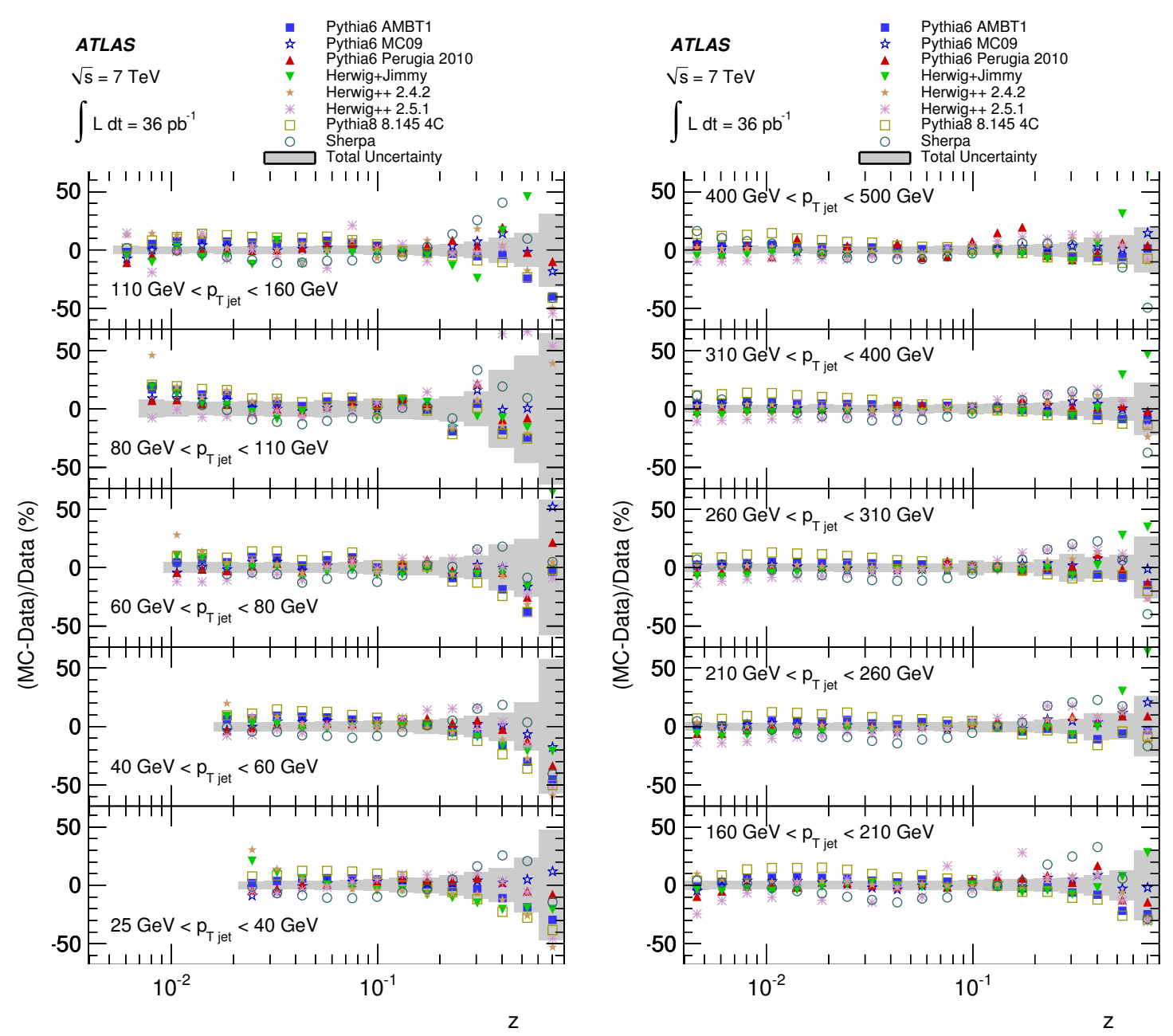

FIGURE 4. The ratio of the charged particle fragmentation function predicted by various Monte Carlo generators to that measured by ATLAS, in different bins of $p_{T \text { jet }}$ [22].

transverse energy $\left(E_{T}^{\gamma}\right)$, in different jet rapidity $\left(y^{\text {jet }}\right)$ ranges and for different photon-jet angular configurations $\left(\eta^{\gamma} y^{\text {jet }} \geq 0, \eta^{\gamma} y^{\text {jet }}<0\right)$ [16]. On the right of Fig.3 the results for one particular $\eta^{\gamma} y^{\text {jet }}$ configuration is shown. The measurement is compared to a NLO prediction, and the theory in general well describes the photon transverse energy spectra with the exception of the low $E_{T}^{\gamma}$ region, a result also observed in the earlier inclusive photon measurement $[17,18]$. ATLAS has also published a measurement of isolated diphoton production [19].

\section{FRAGMENTATION AND JET SHAPES}

At ATLAS several measurements have recently been made to study in depth the "internal" properties of the jets produced in proton-proton collisions at the LHC [20, 21]. In particular, by geometrically associating charged particle tracks to jets reconstructed in 
the calorimeters, the fragmentation function and transverse shape of the charged component of the jets has been measured [22]. Figure 4 shows a comparison of the measured charged particle fragmentation function to the predictions of a variety of different Monte Carlo generators and tunes, in different bins of jet transverse momentum $\left(p_{T}\right.$ jet $)$. No single generator/tune is able to reproduce the data at all values of $p_{T \text { jet }}$.

\section{CONCLUSIONS}

In its first years of operation ATLAS has made a series of measurements in a range of final state topologies that allow for a comprehensive testing of the latest QCD predictions at a new high energy frontier. In general the predictions perform very well, with some interesting exceptions.

\section{REFERENCES}

1. ATLAS Collaboration, JINST 3 (2008) S08003.

2. M. Cacciari, G. P. Salam and G. Soyez, JHEP 0804 (2008) 063.

3. ATLAS Collaboration, Phys. Rev. D 86 (2012) 014022.

4. Z. Nagy, Phys. Rev. D 68 (2003) 094002.

5. S. Alioli, P. Nason, C. Oleari and E. Re, JHEP 1101 (2011) 095.

6. ATLAS Collaboration, ATLAS-CONF-2012-021, http://cdsweb.cern.ch/record/1430730.

7. ATLAS Collaboration, JHEP 1109 (2011) 053.

8. ATLAS Collaboration, Eur. Phys. J. C 71 (2011) 1846.

9. ATLAS Collaboration, Phys. Rev. D 85 (2012) 092002.

10. ATLAS Collaboration, Phys. Rev. D 85 (2012) 032009.

11. ATLAS Collaboration, Phys. Lett. B 707 (2012) 418

12. ATLAS Collaboration, Phys. Lett. B 706 (2012) 295.

13. ATLAS Collaboration, Phys. Lett. B 705 (2011) 415.

14. ATLAS Collaboration, Phys. Rev. D 85 (2012) 012005.

15. F. Landry, R. Brock, P. M. Nadolsky and C. P. Yuan, Phys. Rev. D 67 (2003) 073016.

16. ATLAS Collaboration, Phys. Rev. D 85 (2012) 092014.

17. ATLAS Collaboration, Phys. Lett. B 706 (2011) 150.

18. ATLAS Collaboration, ATL-PHYS-PUB-2011-013, http://cdsweb.cern.ch/record/1395049.

19. ATLAS Collaboration, Phys. Rev. D 85 (2012) 012003.

20. ATLAS Collaboration, JHEP 1205 (2012) 128.

21. ATLAS Collaboration, submitted to Phys. Rev. D, arXiv:1206.5369.

22. ATLAS Collaboration, Eur. Phys. J. C 71 (2011) 1795. 